\title{
White-tailed Deer Food Habits and Nutri- tional Status as Affected by Grazing and Deer-Harvest Management
}

\author{
R.J. WARREN AND L.J. KRYSL
}

\section{Abstract}

White-tailed deer were collected in 1979 and 1980 from two areas in central Texas to determine differences in diets and nutritional status between years, sexes, and areas. Area 1 was more heavily populated with white-tailed deer, exotic big game, and domestic livestock than Area 2. Differences in summer and fall precipitation levels between years were reflected in altered forb and browse consumption by deer as determined from rumen contents. Differences in forb selection, oak mast consumption, and juniper browse consumption were detected between areas and were considered evidence of differences in range condition between areas. Whitetails obtained from Area 1 were older than those from Area 2, but were not significantly larger in carcass weights, which also reflected the lower range condition of Area 1 . Crude protein levels of rumen contents were greater in females than males and were greater in deer obtained from Area 1 than Area 2. These differences in rumen protein resulted from differences in consumption of acorns, a highly preferred, but low protein food item. Kidney fat indices reflected differences in rainfall patterns between years. Native and exotic big game populations and livestock grazing must be controlled to maintain a high level of nutritional status in the economically important white-tailed deer of central Texas.

White-tailed deer (Odocoileus virginianus) represent an important financial asset to most ranchers in central Texas. Monies obtained from hunting leases provide a substantial source of income to many of these landowners (Teer and Forrest 1968). Thus, the proper management of deer herds to maintain optimum nutritional status is important economically. Herds must be kept in balance with the forage resource or overpopulation and malnutrition may result. Some private ranchers risk overpopulated deer herds by not allowing deer populations on their land to be harvested properly.

Numerous studies are available on the responses of white-tailed deer to different grazing practices (McMahan 1964, McMahan and Ramsey 1965, Reardon et al. 1978, Bryant et al. 1981). Kie et al. (1980) examined changes in diet and nutrition with increased herd size of white-tailed deer on the Coastal Bend of Texas. The objective of this study was to compare food habits and nutritional status of deer herds on two areas in central Texas that differed in big game-harvest practices and livestock management.

\footnotetext{
The authors are assistant professor, Department of Range and Wildlife Management, Texas Tech University, Lubbock 79409 , and graduate research assistant, Department of Animal and Range Sciences, New Mexico State University, Las Cruces 88001 . At the time of this research Krysl was research associate, Department of Range and Wildlife Management, Texas Tech University, Lubbock. The cooperation and assistance of D. Harmel, B. Armstrong, and C. Schreiner IV is gratefully acknowledged. The authors thank S. Smith, D. Cox, D. McCelland, V. Wright, and K. Leland for technical assistance. Financial support was provided by the Noxious Brush and Weed Control Program, College of Agricultural Sciences, Texas Tech University and the Caesar Kleberg Foundation for Wildlife Conservation.

This is manuscript number T-9-271, College of Agricultural Sciences, Texas Tech University and is a contribution of the Food Habits Analysis Laboratory in the Department of Range and Wildlife Management.

Manuscript received September, 1981.
}

\section{Study Area and Methods}

Two study areas in Kerr County, Texas, were selected. Rolling topography and stoney soils of limestone origin characteristic of the Edwards Plateau are representative of both areas. Annual precipitation averages $63 \mathrm{~cm}$. The areas were separated by $11.5 \mathrm{~km}$. Each area was surrounded by a 2.3-m deer-proof fence.

Area 1, a privately owned ranch, had about 22,400 ha and was managed primarily for fee-hunting of exotic and native big game. Area 2, a wildlife management area for the Texas Parks and Wildlife Department, had about 2,600 ha and was managed for research and demonstration purposes. Deer hunting on Area 1 has been for trophy bucks only; does have not been hunted since the mid-1960's. No restrictions have been placed on the sex or age of deer harvested on Area 2 since 1957. Annual harvest intensity for the deer herd on Area 1 is about $5-10 \%$ of the population, compared to about $20-25 \%$ on Area 2. White-tailed deer densities on Area 1 are approximately twice as great as those on Area 2. Exotic ungulates, such as axis deer (Axis axis), sika deer(Cervus nippon), fallow deer, (Dama dama), blackbuck antelope (Antilope cervicapra), Barbado sheep (Ovis aries), and aoudad (Ammotragus lervia), are considered "very common" on Area 1, but a re "very ra re" on Area 2. Yearlong grazing of cattle, sheep, and goats in the past has been variable and less controlled on Area 1 than on Area 2. Sheep and goats are not stocked on Area 2, because of dietary competition with white-tailed deer (McMahan 1964, Harmel and Litton 1981). Area 2 has averaged a stocking rate of $1 \mathrm{AU} / 10.9$ ha (Cook 1973). Deferred rotational grazing systems have been used on Area 2, but not on Area 1.

Samples were collected from a total of 109 hunter-killed whitetailed deer during November of 1979 and 1980 . Special permission from the area manager on Area 1 allowed 14 does to be collected and added to the hunter-killed samples from that area. Kidneys with perirenal fat attached, right femurs, and samples of rumen contents were collected from as many of the deer as possible. Each rumen was removed intact and its contents thoroughly handmixed prior to sampling. Two sub-samples were taken randomly from the rumen. The kidney fat index was determined in the field using a triple-beam balance (Riney 1955). Femurs and one 0.5liter sample of rumen material were frozen in the field with dry ice for later analysis. Another 1-liter sample of rumen contents was preserved in $10 \%$ formalin for microhistological examination. Age of deer was estimated by tooth eruption and wear (Severinghaus 1949). Carcass weights were determined and recorded.

Fat content of femur marrow samples was determined by ether extraction (Warren and Kirkpatrick 1978, Kirkpatrick 1980). Crude protein of rumen contents was determined according to A.O.A.C. (1975) methods.

Microscopic slides of reference material and rumen contents were prepared as described by Free et al. (1970). Microhistological examination of rumen material followed procedures outlined by Sparks and Malechek (1968). Five slides were made from each 
sample, and these were examined until 50 fields of view were tallied with identifiable plant fragments. Dietary similarities between selection groups were calculated using Kulczynski's similarity index (Oosting 1956) and Spearman rank-order correlation coefficient (Nie et al. 1975).

Nutritional status data were analyzed by analysis of variance using the general linear model procedure of Barr et al. (1976). A split-plot design was uscd to examine the differences between years, areas, and sexes, as well as all interactions. The significance level used was $P<0.05$.

\section{Results}

White-tailed deer on both areas were primarily browsers rather than grazers during the fall. Browse accounted for $57.1 \%$ and $49.7 \%$ of the fall diet of deer on Area 1 in 1979 and 1980, respectively (Table 1). Oak (Quercus spp.) leaves, Ashe juniper (Juniperus ashei), and acorns comprised the majority of forage consumed by deer on Area 1. Forbs contributed $31.6 \%$ and $42.3 \%$ to the fall diet in 1979 and 1980, respectively, with bladderpods (Lesquerella spp.), spurges (Euphorbia spp.), redseed plantain (Plantago rhodosperma), filaree (Erodium spp.), silverleaf nightshade (Solanum elaegnifolium), and common horehound (Marrubium vulgare) being most important. Texas wintergrass (Stipa leucotricha), a cool-season species, was the only grass consumed substantially during the fall.

Browse comprised $68.6 \%$ and $62.3 \%$ of the fall diet of deer on Area 2 in 1979 and 1980, respectively, with oak mast and leaves being most important (Table 2). Deer on Area 2 consumed more oak mast and less oak leaves and Ashe juniper than deer on Area 1. Forbs contributed $31.6 \%$ and $42.3 \%$ to the fall diet in 1979 and 1980 , respectively, with bladderpods, spurges, globemallows (Sphaeralcea spp.), whorled nodviolet (Hybanthus verticillatus), and filaree being most important. Deer on Area 2 consumed a much greater variety of forb species than deer on Area 1 (27 vs. 18,

Table 1. Percent relative frequency of plant fragments in fall 1979 and 1980 deer diets on Area 1.

\begin{tabular}{|c|c|c|c|c|}
\hline \multirow[b]{2}{*}{ Species } & \multirow[b]{2}{*}{$\begin{array}{c}1979 \text { Total } \\
(15)^{\prime}\end{array}$} & \multirow[b]{2}{*}{$\begin{array}{l}1980 \text { Total } \\
\text { (40) }\end{array}$} & \multicolumn{2}{|c|}{1980} \\
\hline & & & $\begin{array}{c}\text { Males } \\
(32)\end{array}$ & $\begin{array}{c}\text { Females } \\
(8)\end{array}$ \\
\hline \multicolumn{5}{|l|}{ Grasses: } \\
\hline Texas wintergrass (Stipa leucotricha) & 2.0 & 1.6 & 1.9 & 1.2 \\
\hline Sedge (Carex spp.) & $T_{r^{2}}$ & $\operatorname{Tr}$ & $\operatorname{Tr}$ & $\operatorname{Tr}$ \\
\hline Sideoats grama (Bouteloua curtipendula) & - & $\mathrm{Tr}$ & $\mathrm{Tr}$ & - \\
\hline Bristlegrass (Setaria spp.) & $\operatorname{Tr}$ & $\operatorname{Tr}$ & $\mathrm{Tr}$ & $\operatorname{Tr}$ \\
\hline Threeawns (Aristida spp.) & $\underline{-}$ & Tr & $\mathrm{Tr}$ & - \\
\hline Blue grama (Bouteloua gracilis) & $\operatorname{Tr}$ & $\mathrm{Tr}$ & $\mathrm{Tr}$ & - \\
\hline Rescuegrass (Bromus catharticus) & 1.0 & $\mathrm{Tr}$ & $\mathrm{Tr}$ & - \\
\hline Panic grass (Panicum spp.) & - & $\mathrm{Tr}$ & $\mathrm{Tr}$ & 1.2 \\
\hline Unknown grasses & $\operatorname{Tr}$ & $\mathrm{Tr}$ & $\operatorname{Tr}$ & - \\
\hline Total grasses & 6.2 & 4.3 & 5.3 & 3.2 \\
\hline \multicolumn{5}{|l|}{ Forbs: } \\
\hline Bladderpods (Lesquerella spp.) & 17.1 & 28.6 & 14.1 & 42.2 \\
\hline Spurges (Euphorbia spp.) & 5.0 & 3.5 & 3.9 & 3.1 \\
\hline Fleabane (Erigeron spp.) & $\mathrm{Tr}$ & 1.0 & 1.9 & $\mathrm{Tr}$ \\
\hline Redseed plaintain (Plantago rhodosperma) & 2.0 & 1.6 & 1.5 & 1.6 \\
\hline Filaree (Erodium spp.) & 1.8 & 1.2 & 1.4 & $\mathrm{Tr}$ \\
\hline Primrose (Oenothera spp.) & $\operatorname{Tr}$ & $\operatorname{Tr}$ & 1.1 & $\operatorname{Tr}$ \\
\hline Silverleaf nightshade (Solamum elaegnifolium) & 2.1 & 1.0 & 1.1 & $\mathrm{Tr}$ \\
\hline Globemallows (Sphaeralcea spp.) & $\operatorname{Tr}$ & $\operatorname{Tr}$ & 1.0 & $\operatorname{Tr}$ \\
\hline White sage (Artemisia ludoviciana) & Tr & $\operatorname{Tr}$ & 1.0 & - \\
\hline Common horehound (Marrubium vulgare) & $\operatorname{Tr}$ & 1.9 & 1.0 & 2.7 \\
\hline Puccoon (Lithospermum spp.) & $\operatorname{Tr}$ & $\operatorname{Tr}$ & $\mathrm{Tr}$ & $\mathrm{Tr}$ \\
\hline Zinnia (Zinnia spp.) & Tr & $\mathrm{Tr}$ & $\mathrm{Tr}$ & $\mathrm{Tr}$ \\
\hline Groundcherry (Physalis spp.) & 1.1 & $\mathrm{Tr}$ & $\mathrm{Tr}$ & $\mathrm{Tr}$ \\
\hline Locoweed (Astragulas spp.) & $\mathbf{T r}_{\mathbf{r}}$ & $\mathrm{Tr}$ & $\mathrm{Tr}$ & - \\
\hline Milkwort (Polygala spp.) & - & $\mathrm{Tr}$ & $\operatorname{Tr}$ & - \\
\hline Ragweed (Ambrosia spp.) & - & $\mathrm{Tr}$ & - & $\overline{T r}$ \\
\hline Sida (Sida spp.) & - & $\mathrm{Tr}$ & - & $T_{r}$ \\
\hline Pricklepoppy (Argemone spp.) & - & $\operatorname{Tr}$ & - & $\operatorname{Tr}$ \\
\hline Total forbs & 31.6 & 42.3 & 29.9 & 54.6 \\
\hline \multicolumn{5}{|l|}{ Browse: } \\
\hline Oak leaves (Quercus spp.) & 19.8 & 22.5 & 27.5 & 17.4 \\
\hline Ashe juniper (Juniperus ashei) & 26.2 & 17.1 & 18.5 & 15.6 \\
\hline Oak mast (Quercus spp.) & 9.1 & 5.4 & 8.4 & 2.4 \\
\hline Sumac (Rhus spp.) & $\mathrm{Tr}$ & $\mathrm{Tr}$ & 1.3 & 2.4 \\
\hline Acacia (Acacia spp.) & - & $\mathrm{Tr}$ & 1.2 & - \\
\hline Elbowbush (Forestiera spp.) & - & $\mathrm{Tr}$ & 1.0 & - \\
\hline Cacti (Opuntia spp.) & 1.2 & $\mathrm{Tr}$ & 1.0 & $\mathrm{Tr}$ \\
\hline Baccharis (Baccharis spp.) & - & $\mathrm{Tr}$ & Tr & - \\
\hline Algerita (Berberis trifolata) & - & $\mathrm{Tr}$ & $\operatorname{Tr}$ & $\operatorname{Tr}$ \\
\hline Mesquite (Prosopis chilensis) & $\operatorname{Tr}$ & 1.6 & $\mathrm{Tr}$ & 2.5 \\
\hline Total Browse & 57.1 & 49.7 & 60.2 & 39.4 \\
\hline Unknown (Browse or Forb) & 5.1 & 3.7 & 4.6 & 2.8 \\
\hline Grand Total & 100.0 & 100.0 & 100.0 & 100.0 \\
\hline
\end{tabular}


respectively). Texas wintergrass also was the major grass species used by deer on Area 2.

Similarity indices and rank-order correlation coefficients (Table 3) indicated that deer diets within areas and between 1979 and 1980 were similar. Further comparisons were made with 1980 data only, because of the smaller sample size in 1979. These comparisons indicated that diets within areas and between male and female deer were similar. However, deer diets were substantially different between areas. Female diets were least similar between areas $(31.5 \%)$, primarily because females on Area late large quantities of bladderpods (42.2\%), while females on Area 2 consumed primarily oak mast $(50.7 \%)$.
Deer collected from Area 1 were older than deer collected from Area $2(\bar{X}=4.7$ years vs. 2.6 years, respectively; Tables 4 and 5$)$. Carcass weights (Tables 4 and 5) were significantly different between male and female deer $(\bar{X}=36.4 \mathrm{~kg}$ vs. $23.6 \mathrm{~kg}$, respectively). Females had greater levels of crude protein in their rumina (Tables 4 and 5 ) than males ( $\bar{X}=8.7 \%$ vs. $6.6 \%$, respectively). Additionally, deer on Area 1 had greater levels of rumen protein than those on Area $2(\vec{x}=8.5 \%$ vs. $6.3 \%$, respectively). Kidney fat indices (Tables 4 and 5) determined for all deer in 1979 were significantly greater than in $1980 \mathrm{X}=77.7 \%$ vs. $19.7 \%$, respectively). No significant differences in femur marrow fat content (Tables 4 and 5) were detected between years, sexes, or areas.

Table 2. Percent relative frequency of plant fragments in fall 1979 and 1980 deer diets on Area 2.

\begin{tabular}{|c|c|c|c|c|}
\hline \multirow[b]{2}{*}{ Species } & \multirow[b]{2}{*}{$\begin{array}{l}1979 \text { total } \\
(13)^{1}\end{array}$} & \multirow[b]{2}{*}{$\begin{array}{l}1980 \text { total } \\
(60)\end{array}$} & \multicolumn{2}{|c|}{1980} \\
\hline & & & $\begin{array}{c}\text { Males } \\
(48)\end{array}$ & $\begin{array}{l}\text { Females } \\
\text { (12) }\end{array}$ \\
\hline Grasses: & & & & \\
\hline $\begin{array}{l}\text { Texas wintergrass (Stipa leucotricha) } \\
\text { Plains lovegrass (Eragrostis intermedia) } \\
\text { Sideoats grama (Bouteloua curtipendula) } \\
\text { Rescuegrass (Bromus catharticus) } \\
\text { Bristlegrass (Setaria spp.) } \\
\text { Threeawns (Aristida spp.) } \\
\text { Panic grass (Panicum spp.) } \\
\text { Unknown grasses } \\
\quad \text { Total Grasses }\end{array}$ & $\begin{array}{l}2.1 \\
1.0 \\
\operatorname{Tr} \\
1.0 \\
\overline{-} \\
\overline{\operatorname{Tr}} \\
\overline{4.9}\end{array}$ & $\begin{array}{l}3.9 \\
\operatorname{Tr} r^{2} \\
\operatorname{Tr} \\
\operatorname{Tr} \\
\operatorname{Tr} \\
\operatorname{Tr} \\
\operatorname{Tr} \\
\operatorname{Tr} \\
5.4\end{array}$ & $\begin{array}{l}2.6 \\
\operatorname{Tr} \\
\operatorname{Tr} \\
\operatorname{Tr} \\
\operatorname{Tr} \\
\operatorname{Tr} \\
\overline{T r} \\
3.9\end{array}$ & $\begin{array}{l}5.1 \\
\overline{\operatorname{Tr}} \\
\operatorname{Tr} \\
- \\
\overline{\operatorname{Tr}} \\
\operatorname{Tr} \\
6.9\end{array}$ \\
\hline $\begin{array}{l}\text { Forbs: } \\
\text { Bladderpods (Lesquerella spp.) } \\
\text { Spurges (Euphorbia spp.) } \\
\text { Globemallows (Sphaeralcea spp.) } \\
\text { White sage (Artemisia ludoviciana) } \\
\text { Silverleaf nightshade (Solanum elaegnifolium) } \\
\text { Fleabane (Erigeron spp.) } \\
\text { Whorled nodviolet (Hybanthus verticillatus) } \\
\text { Primrose (Oenothera spp.) } \\
\text { Common horehound (Marrubium vulgare) } \\
\text { Locoweed (Astragulas spp.) } \\
\text { Flax (Linum spp.) } \\
\text { Filaree (Erodium spp.) } \\
\text { Croton (Croton spp.) } \\
\text { Sand-lily (Mentzelia spp.) } \\
\text { Arrowleaf sida (Sida filicaulis) } \\
\text { Groundsel (Senecio spp.) } \\
\text { Groundcherry (Physalis spp.) } \\
\text { Flannel mullien (Verbascum thapsus) } \\
\text { Common ragweed (Ambrosia psilostachya) } \\
\text { Carlesss weed (Amaranthus graecizans) } \\
\text { Coneflower (Ratibida columnaris) } \\
\text { Zinnia (Zinnia spp.) } \\
\text { Milkwort (Polygala spp.) } \\
\text { Redseed plantain (Plantago rhodosperma) } \\
\text { Puccoon (Lithospermum spp.) } \\
\text { Sage (Saliva farinacea) } \\
\text { Buckwheat (Eriogonum spp.) } \\
\text { Total Forbs }\end{array}$ & $\begin{array}{l}14.2 \\
2.0 \\
1.0 \\
\operatorname{Tr} \\
1.0 \\
\operatorname{Tr} \\
2.4 \\
1.1 \\
\operatorname{Tr} \\
\operatorname{Tr} \\
\operatorname{Tr} \\
2.0 \\
- \\
- \\
- \\
- \\
\operatorname{Tr} \\
- \\
\overline{T r} \\
- \\
\overline{T r} \\
\operatorname{Tr} \\
- \\
- \\
\overline{-} \\
24.0\end{array}$ & $\begin{array}{c}10.6 \\
2.3 \\
2.8 \\
1.2 \\
\mathrm{Tr} \\
\mathrm{Tr} \\
1.4 \\
1.3 \\
1.1 \\
\mathrm{Tr} \\
\mathrm{Tr} \\
1.1 \\
\mathrm{Tr} \\
\mathrm{Tr} \\
\mathrm{Tr} \\
\mathrm{Tr} \\
\mathrm{Tr} \\
\mathrm{Tr} \\
\mathrm{Tr} \\
\mathrm{Tr} \\
\mathrm{Tr} \\
\mathrm{Tr} \\
\mathrm{Tr} \\
\mathrm{Tr} \\
\mathrm{Tr} \\
\mathrm{Tr} \\
\mathrm{Tr} \\
29.3\end{array}$ & $\begin{array}{c}9.2 \\
3.1 \\
2.4 \\
1.6 \\
1.2 \\
1.1 \\
1.0 \\
1.0 \\
\mathrm{Tr} \\
\mathrm{Tr} \\
\mathrm{Tr} \\
\mathrm{Tr} \\
\mathrm{Tr} \\
\mathrm{Tr} \\
\mathrm{Tr} \\
\mathrm{Tr} \\
\mathrm{Tr} \\
\mathrm{Tr} \\
\mathrm{Tr} \\
\mathrm{Tr} \\
\mathrm{Tr} \\
\mathrm{Tr} \\
\mathrm{Tr} \\
\mathrm{Tr} \\
\mathrm{Tr} \\
- \\
- \\
27.0\end{array}$ & $\begin{array}{l}12.0 \\
1.4 \\
3.2 \\
\mathrm{Tr} \\
\mathrm{Tr} \\
\mathrm{Tr} \\
1.8 \\
1.5 \\
1.5 \\
\mathrm{Tr} \\
\overline{1.5} \\
1.3 \\
\overline{\mathrm{Tr}} \\
\overline{1.2} \\
\mathrm{Tr} \\
\mathrm{Tr} \\
\mathrm{Tr} \\
\overline{-} \\
\overline{-} \\
\overline{\mathrm{Tr}} \\
\overline{\mathrm{Tr}} \\
\mathrm{Tr} \\
31.6\end{array}$ \\
\hline $\begin{array}{l}\text { Browse: } \\
\text { Oak mast (Quercus spp.) } \\
\text { Oak leaves (Quercus spp.) } \\
\text { Ashe juniper (Juniperus ashei) } \\
\text { Woollybucket bumelia (Bumelia lanuginosa) } \\
\text { Grape (Prunus spp.) } \\
\text { Sumac (Rhus spp.) } \\
\text { Cacti (Opuntia spp.) } \\
\text { Mesquite (Prosopis chilensis) } \\
\text { Total browse }\end{array}$ & $\begin{array}{c}55.1 \\
10.2 \\
2.1 \\
\operatorname{Tr} \\
- \\
\overline{T r} \\
\operatorname{Tr} \\
68.6\end{array}$ & $\begin{array}{l}54.5 \\
5.8 \\
\operatorname{Tr} \\
\operatorname{Tr} \\
\operatorname{Tr} \\
\operatorname{Tr} \\
\operatorname{Tr} \\
\operatorname{Tr} \\
62.3\end{array}$ & $\begin{array}{c}58.3 \\
6.1 \\
\mathrm{Tr} \\
\mathrm{Tr} \\
\mathrm{Tr} \\
\mathrm{Tr} \\
\mathrm{Tr} \\
- \\
66.1\end{array}$ & $\begin{array}{c}50.7 \\
5.5 \\
\mathrm{Tr} \\
- \\
\mathrm{Tr} \\
\mathrm{Tr} \\
\mathrm{Tr} \\
\mathrm{Tr} \\
58.5\end{array}$ \\
\hline Unknown (Browse or Forb) $\quad$ Grand Total & $\begin{array}{r}2.5 \\
100.0\end{array}$ & $\begin{array}{r}3.0 \\
100.0\end{array}$ & $\begin{array}{r}3.0 \\
100.0\end{array}$ & $\begin{array}{r}3.0 \\
100.0\end{array}$ \\
\hline
\end{tabular}

'Sample size in parentheses.

$2 \mathrm{Tr}=\operatorname{traces}(<1.0 \%$ R.F. $)$. 
Table 3. Similarity indices (\%) and Spearman rank-order correlation coefficients $\left(r_{\mathrm{a}}\right)$ of selected dietary comparisons.

\begin{tabular}{lcc}
\hline \hline Comparison & $\%$ & $r_{\mathbf{s}}$ \\
\hline Area 1 (1979) vs. Area 1 (1980) & 82.1 & 0.77 \\
Area 2 (1979) vs. Area 2 (1980) & 87.0 & 0.85 \\
Males (1980, Area 1) vs. Females (1980, Area 1) & 65.7 & 0.76 \\
Males (1980, Area 2) vs. Females (1980, Area 2) & 84.4 & 0.70 \\
Males (1980, Area 1) vs. Males (1980, Area 2) & 40.1 & 0.27 \\
Females (1980, Area 1) vs. Females (1980, Area 2) & 31.5 & 0.57 \\
Area 1 (1980) vs. Area 2 (1980) & 39.7 & 0.49 \\
\hline
\end{tabular}

\section{Discussion}

Possible biases inherent in the microscopic analysis of plant fragments as performed in this study were discussed by Kie et al. (1980). Absolute dietary percentages of bladderpods and silverleaf nightshade may have been overestimated by this technique. However, conclusions regarding relative changes in dietary components between years, sexes, and areas can be made.

Food habits of deer collected in this study varied between years, perhaps as a result of different rainfall patterns. The amount of forbs in the diet of deer from both areas (Tables 1 and 2) was greater in 1980 than in 1979. This observation may be the result of substantially greater fall precipitation in 1980 compared to 1979 (Table 6). Furthermore, browse apparently was more important to deer in 1979 than in 1980 . Substantially more spring and summer precipitation occurred in 1979 than in 1980 and may explain this observation. One other consistent trend in food habits observed between years for both areas was that Ashe juniper appeared to be more important in 1979 than in 1980. The lower fall rainfall in 1979 than in 1980 may have forced the deer to rely more heavily on juniper browse. Juniper is considered a low-palatability, emergency browse for white-tailed deer (Hill 1946).
Table 6. Average seasonal and annual rainfall (cm) at Area 2 during 1979, 1980, and over a 27 year period (1952-1979).1

\begin{tabular}{lcrc}
\hline \hline Season & $\begin{array}{r}1979 \\
(\mathrm{~cm})\end{array}$ & $\begin{array}{c}1980 \\
(\mathrm{~cm})\end{array}$ & $\begin{array}{c}1952-1979 \\
(\mathrm{~cm})\end{array}$ \\
\hline Winter (Dec.-Feb.) & 11.5 & 9.4 & 8.7 \\
Spring (Mar.-May) & 20.5 & 12.2 & 15.7 \\
Summer (June-Aug.) & 26.5 & 8.5 & 19.1 \\
Fall (Sept.-Nov.) & 5.0 & 38.9 & 20.0 \\
Annual & 63.5 & 69.0 & 63.5 \\
\hline
\end{tabular}

'Data obtained from Harmel and Litton (1981) and personal communications with area managers at Area 2.

Differences in deer diets between areas also were observed. It was assumed that Area 2 was managed better than Area 1, based on differences in deer populations, exotic ungulate populations, and grazing practices. Yet, in both years deer collected from Area 2 had a substantially lower amount of forbs in their rumina than deer from Area 1 (Tables 1 and 2). Most literature (McMahan 1964, Bryant et al. 1981) indicates that poor range management will reduce the amount of forbs in the diet of white-tailed deer. The observed differences in forb selection by deer in this study are probably the result of differences in consumption of highly preferred acorns. Deer on Area 2 consumed substantially more acorns than deer on Area 1, which probably reduced their consumption of forbs. Harlow et al. (1975) demonstrated that deer consume lower amounts of herbaceous plants in years of oak mast abundance. Furthermore, deer on Area 2 consumed a much wider variety of forb species than those on Area 1. Forb diversity in the Edwards Plateau is much greater on pastures in excellent range condition than on pastures in poor range condition (F.C. Bryant, pers. comm.). In addition, deer on Area 2 consumed much less juniper than deer on Area 1. Bryant et al. (1981) reported that deer on pastures in poor range condition consumed juniper, whereas deer

Table 4. Mean ( $\bar{x} \pm S E)$ age and indices of nutritional status for white-tailed deer obtained from Area 1.

\begin{tabular}{llcccccc}
\hline \hline Year & Sex & $\begin{array}{c}\text { Sample } \\
\text { size }\end{array}$ & $\begin{array}{c}\text { Age } \\
(\text { years) }\end{array}$ & $\begin{array}{c}\text { Carcass weight } \\
(\mathrm{kg})^{2}\end{array}$ & $\begin{array}{c}\text { Rumen protein } \\
(\%)^{3}\end{array}$ & $\begin{array}{c}\text { Kidney fat index } \\
(\%)^{4}\end{array}$ & $\begin{array}{c}\text { Femur marrow fat } \\
(\%)^{5}\end{array}$ \\
\hline 1979 & Male & 8 & $3.9 \pm 0.6$ & $36.2 \pm 1.5^{6}$ & $10.2 \pm 0.5^{6}$ & $98.4 \pm 21.5^{6}$ & $80.8 \pm 4.8$ \\
1979 & Female & 7 & $4.9 \pm 1.2$ & $18.2 \pm 1.9$ & $10.4 \pm 0.6$ & $15.3 \pm 4.7$ & $56.9 \pm 11.4$ \\
1980 & Male & 29 & $4.8 \pm 0.2^{6}$ & $41.8 \pm 0.9$ & $6.9 \pm 0.4^{7}$ & $22.9 \pm 2.0$ & $88.5 \pm 1.3^{8}$ \\
1980 & Female & 6 & $5.0 \pm 0.8$ & $26.0 \pm 2.3$ & $11.6 \pm 1.7$ & $21.8 \pm 6.0$ & $91.2 \pm 1.5$ \\
\hline
\end{tabular}

${ }^{1}$ Analysis of age data revealed a significant $(\boldsymbol{P}<0.05)$ area effect. and sex $\times$ area interaction.

2 Analysis of carcass weight data revealed a significant $(P<0.05)$ sex effect and year $\times$ area interaction.

${ }_{3}^{3}$ Analysis of rumen protein data revealed a significant $(P<0.05)$ sex effect, area effect, and year $\times$ sex $\times$ area interaction.

4Analysis of kidney fat index data revealed a significant $(P<0.05)$ year effect, year $X$ sex interaction, and year $X$ sex $X$ area interaction.

${ }^{s}$ Analysis of femur marrow fat data revealed a significant $(P<0.05)$ year $X$ sex interaction and year $X$ sex $X$ area interaction.

-Sample size for this mean is one less than indicated.

'Sample size for this mean is 27.

${ }^{8}$ Sample size for this mean is 18 .

Table 5. Mean ( $\mathbf{x} \pm$ SE) age and indices of nutritional status for white-tailed deer obtalned from Area 2.

\begin{tabular}{llcccccc}
\hline \hline Year & Sex & $\begin{array}{c}\text { Sample } \\
\text { size }\end{array}$ & $\begin{array}{c}\text { Age } \\
(\text { years) }\end{array}$ & $\begin{array}{c}\text { Carcass weight } \\
(\mathrm{kg})^{2}\end{array}$ & $\begin{array}{c}\text { Rumen protein } \\
(\%)^{3}\end{array}$ & $\begin{array}{c}\text { Kidney fat index } \\
(\%)^{4}\end{array}$ & $\begin{array}{c}\text { Femur marrow fat } \\
(\%)^{5}\end{array}$ \\
\hline 1979 & Male & 10 & $1.9 \pm 0.2$ & $34.4 \pm 1.9$ & $5.8 \pm 0.5$ & $109.7 \pm 17.9$ & $81.0 \pm 2.3$ \\
1979 & Female & 3 & $3.5 \pm 2.1$ & $22.7 \pm 2.0$ & $7.2 \pm 0.4$ & $68.0 \pm 19.1$ & $86.1 \pm 6.9$ \\
1980 & Male & 44 & $2.5 \pm 0.2$ & $33.2 \pm 1.4^{6}$ & $6.1 \pm 0.37$ & $17.1 \pm 1.8$ & $81.5 \pm 1.59$ \\
1980 & Female & 15 & $3.2 \pm 0.7$ & $25.3 \pm 1.9$ & $6.9 \pm 0.56$ & $18.6 \pm 3.16$ & $71.0 \pm 4.110$ \\
\hline
\end{tabular}

IAnalysis of age data revealed a signifcant $(P<0.05)$ area effect and sex $\times$ area interaction.

${ }^{2}$ Analysis of carcass weight data revealed a significant $(R<0.0)$ sex effect and year $\times$ area interaction.

${ }^{3}$ Analysis of rumen protein data revealed a significant $(P<0.05)$ sex effect, area effect, and year $X$ sex area interaction.

4Analysis of kidney fat index data revealed a significant $(P<0.05)$ year effect, year $\times \times$ sex interaction, and year $\times$ sex $X$ area interaction.

${ }^{5}$ Analysis of femur marrow fat data revealed a significant $(P<0.05)$ year $X$ sex interaction and year $X$ sex $X$ interaction.

'Sample size for this mean is one less than indicated.

'Sample size for this mean is 41 .

${ }^{8}$ Sample size for this mean is 37 .

${ }^{9}$ Sample size for this mean is 30 .

10Sample size for this mean is 13 . 
on pastures in excellent range condition did not select juniper. These differences in deer diets between areas indicate that the pastures on Area 2 were in better range condition than those on Area 1.

A bias in animal collection techniques probably accounted for the significant difference detected between areas for deer age (Tables 4 and 5). All males killed by hunters on Area 1 were selected as trophy animals, based primarily on antler characteristics. Thus, these animals would be expected to be older. Furthermore, older deer should weigh more than younger deer (Short et al. 1969, Knowlton et al. 1979). Deer obtained from Area 1 averaged 2 years older than deer from Area 2, but did not exhibit significantly greater carcass weights (Tables 4 and 5). These observations suggest that deer on Area 1 were of a lower nutritional status than deer on Area 2, probably as a result of range condition differences.

The population age structures also may have differed between areas. Short (1972) determined that heavy hunting produces a young population age structure. Light hunting pressure will produce an old age structure in the population. Thus, the light hunting pressure that occurred on Area 1 may have produced a deer herd with an older age structure than found on Area 2, which is heavily hunted.

The higher dietary crude protein in females (Tables 4 and 5) than males during this study probably reflected behavioral differences (i.e., rutting by males) rather than differences in nutritional needs. Males are known to alter their feed intake during the fall rut (Warren et al. 1981). Also, males ate more acorns than femalcs (Tables 1 and 2). Acorns are low in crude protein and high in energy content (Short 1971, Harlow et al. 1975). Thus, the higher acorn content in rumina of males would have reduced the rumen protein levels, as compared to females.

Rumen protein content also was greater in deer from Area 1 than Area 2 (Tables 4 and 5). This apparent anomalous result can be explained by the fact that deer from Area 2 consumed substantially more oak mast than deer from Area 1 (Tables 1 and 2). The higher acorn content in the diets of deer from A rea 2 would have reduced rumen protein levels and does not necessarily indicate a lower forage quality on Area 2. The lower amount of acorns consumed by white-tailed deer on Area 1, compared to A rea 2, may be due to the greater numbers of ungulates competing for the available acorns on Area 1. Sheep and goats readily consume oak mast (McMahan 1964), as do axis deer, sika deer, fallow deer, blackbuck antelope, and aoudad (Butts et al. 1976). It also is possible that mast availability may have differed between areas. Overpopulated deer herds can decrease hardwood forest regeneration (Graham 1954, Webb et al. 1956).

Lower kidney fat indices (Tables 4 and 5) observed in 1980 , compared to 1979, are probably the result of below normal summer precipitation in 1980 (Table 6). Summer rainfall in 1979 was above normal. Thus, these data indicate that a delay of approximately 3 months would be required before a significant change in rainfall would be reflected in kidney fat levels. Kidney fat is a widely accepted nutritional index, although the temporal responsiveness of kidney fat to altered nutritional conditions is not well documented. Warren and Kirkpatrick (1982) conducted a controlled nutritional experiment in which white-tailed deerfawns were placed on high energy $(2,938 \mathrm{kcal}$ digestible energy $/ \mathrm{kg})$ and low energy $(2,336 \mathrm{kcal}$ digestible energy $/ \mathrm{kg})$ diets for 4 weeks. Kidney fat levels were significantly lower in fawns on low energy diets than in those on high energy diets. Additional studies are needed to fully elucidate the responsiveness of kidney fat in adult deer.

No differences in femur marrow fat content were detected in this study (Tables 4 and 5). Fat reserves are catabolized in an ordered, sequential manner (Harris 1945, Riney 1955, Dauphine 1971). Femur marrow fat is one of the last fat reserves to be used by deer, and is not expected to decrease substantially until kidney fat indices drop below 30\% (Ransom 1965, Warren and Kirkpatrick 1982). Thus, deer in this study were not to the point nutritionally at which femur marrow fat would be used.

\section{Conclusion}

Data obtained in this study indicated that intensive white-tailed deer harvests, low exotic ungulate populations, and controlled livestock grazing improve the nutritional status of white-tailed deer populations in the Edwards Plateau of Texas. Nutritional differences between areas were apparent, but somewhat obscured by significant differences in ages of collected deer. Deer collected from the lightly populated area attained a level of nutritional status not significantly different from that of deer on the densely populated area, but at a much earlier age. Nutritional differences between areas probably would have been more obvious if the ages of collected deer were similar, and if the collections had been conducted at a more nutritionally st ressful season (e.g., February).

\section{Literature Cited}

A.O.A.C. 1975. Official methods of analysis of the Association of Analytical Chemists. 12th Ed. Washington, D.C.

Barr, A.J., J.H. Goodnight, J.S. Sall, J.T. Helwig. 1976. A user's guide to S.A.S. 76. SAS Institute Inc., Raleigh, N.C.

Bryant, F.C., C.A. Taylor, and L.B. Merrill. 1981. White-tailed deer diets from pastures excellent and poor range condition. J. Range Manage. 34:193-200.

Butts, G.L., M.J. Anderegg, W.E. Armstrong, D.E. Harmel, C.W. Ramsey, and S.H. Sorola. 1976. Food habits of five exotic ungulates on Kerr Wildlife Management Area, Texas. Texas Parks Wildl. Dep. Tech. Ser. 30.

Cook, R.L. 1973. Management implications of heavy hunting pressure on Texas white-tailed deer on the Kerr Wildlife Management Area. Proc. Ann. Conf. S.E. Assoc. Fish Wildl. Agencies 27:114-119.

Dauphine, T.C., Jr. 1971. Physical variables as index to condition on barren-ground caribou. Trans. Northeast. Wild. Soc. 28:91-108.

Free, J.C., R.M. Hansen, and P.L. Sims. 1970. Estimating dry weights of food plants in feces of herbivores. J. Range Manage. 23:300-302.

Graham, S.A. 1954. Changes in northern Michigan forests from browsing by deer. Trans. North Amer. Wildl. Nat. Res. Conf. 19:526-533.

Harlow, R.F., J.B. Whelan, H.S. Crawford, and J.E. Skeen. 1975. Deer foods during years of oak mast abundance and scarcity. J. Wildl. Manage. 39:330-336.

Harmel, D.E., and G.W. Litton. 1981. Deer management in the Edwards Plateau of Texas. Texas Parks Wildl. Dep. Booklet 7000-86.

Harris, D. 1945. Symptoms of malnutrition in deer. J. Wildl. Manage. 9:319-322.

Hill, R.R. 1946. Palatability ratings of Black Hills plants for white-tailed deer. J. Wildl. Manage. 10:47-54.

Kie, J.G., D.L. Drawe, and G. Scott. 1980. Changes in diet and nutrition with increased herd size in Texas white-tailed deer. J. Range Manage. 33:28-34

Kirkpatrick, R.L. 1980. Physiological indices in wildlife management, p. 99-112. In: S.D. Schemnitz (ed.), Wildlife Management Techniques Manual. 4th ed. The Wildlife Society, Washington, D.C.

Knowlton, F.F., M. White, and J.G. Kie. 1979. Weight patterns of wild white-tailed deer in southern Texas. The Welder Wildlife Foundation Research Program: The First 22 Years, a Symposium. Corpus Christi, Texas. October 14, 1978.

McMahan, C.A. 1964. Comparative food habits of deer and three classes of livestock. J. Wildl. Manage. 28:798-808.

McMahan, C.A., and C.W. Ramsey. 1965. Response of deer and livestock to controlled grazing in central Texas. J. Range Manage. 18:1-7.

Nie, N.H., C.H. Hull, J.G. Jenkins, K. Steinbrenner, and D.H. Bent. 1975. Statistical Package for the Social Sciences. 2nd ed. McGraw-Hill Book Co., New York.

Oosting, H.J. 1956. The study of plant communities. Witt. Freeman and Co., San Francisco.

Ransom, A.B. 1965. Kidney and marrow fat as indicators of white-tailed deer condition. J. Wildl. Manage. 29:397-398.

Reardon, P.O., L.B. Merrill, and C.A. Taylor, J. 1978. White-tailed deer preferences and hunter success under various grazing systems. J. Range Manage. 31:40-42.

Riney, T. 1955. Evaluating condition of free-ranging red deer (Cervus elaphus), with special reference to New Zealand. New Zealand J. Sci. Technol. 36:429-463.

Severinghaus, C.W. 1949. Tooth development and wear as criteria of age in white-tailed deer. J. Wildl. Manage. 13:195-216.

Short, H.L. 1971. Forage digestibility and diet of deer on southern upland range. J. Wildl. Manage. 35:698-706. 
Short, H.L. 1972. Ecological framework for deer management. J. Forest. 70:200-203.

Short, H.L., J.D. Newsom, G.L. McCoy, and J.F. Fowler. 1969. Effects of nutrition and climate on southern deer. Trans. North Amer. Wildl. Nat. Rex. Conf. 34:137-145.

Sparks, D.R., and J.C. Malechek. 1968. Estimating percentage dry weight in diets using a microscopic technique. J. Range Manage. 21:264-265.

Teer, J.G., and N.K. Forrest. 1968. Bionomic and ethical implications of commercial game harvest programs. Trans. North Amer. Wild. Nat. Res. Conf. 33:192-204
Warren, R.J., and R.L. Kirkpatrick. 1978. Indices of nutritional status in cottontail rabbits fed controlled diets. J. Wildl. Manage. 42:154-158.

Warren, R.J., and R.L. Kirkepatrick. 1982. Evaluating nutritional status of white-tailed deer using fat indices. Proc. Ann. Conf. S.E. Assoc. Fish Wildl. Agencies 36: (In Press).

Warren, R.J., R.L. Kirkpatrick. A. Oelschlaeger, P.F. Scanlon, and F.C. Gwazdauskas. 1981. Dietary and seasonal influences on nutritional indices of adult male white-tailed deer. J. Wildl. Manage. 45:926-936.

Webb, W.L., R.T. King, and E.F. Patric. 1956. Effect of the white-tailed deer on mature northern hardward forest. J. Forest. 54:391-398.

\section{In Appreciation for Professional Contributions}

Papers submitted to the Journal of Range Management are processed and evaluated by appointed associate editors and an editor. Supporting them are many reviewers, chosen for their specialized qualifications. The names of the associate editors are given in the inside front cover; the names of those who have contributed time and thought as reviewers are given below. Both groups deserve sincerest thanks from the Society and the profession for their unselfish efforts to maintain and improve the quality of papers in the Journal.

Every paper contributed to the Journal of Range Management is reviewed by at least two scientifically qualified persons. This represents no small burden and thus no small contribution from those who have participated in review and editing process. On behalf of all Society members, thanks to each of them.

Alden, E.

Allison, $\mathbf{C}$.

Allred, $\mathbf{K}$.

Anderson, B. E.

Arno, S. F.

Arthur, J., III

Bahre, C. J.

Bailey, A. W.

Barbour, M.

Barrett, R. H.

Bartlett, E. T.

Bartlett, T. T.

Bartolome, J.

Bartos, D. L.

Beaton, J.

Beatty, E. R.

Bjugstad, A.

Blackburn, W.

Blair, R.

Bonham, C. D.

Bovey, R. W.

Brady, W.

Bragg, T. B.

Briske, D.

Britton, C. M.

Brock, J.

Brown, R. W.

Bryant, $F$.

Buckhouse, J.

Bucks, D.

Bunting, $S$.

Burns, J. C.

Cain, B.

Carpenter, L. H.

Case, R. M.

Clark, D.

Clary, W. P.

Cole, C. V.

Cox, J.

Coyne, P. I.

Currie, P. O.

Dahl, W. E.
Denham, A. H

Dietz, D.

Donart, G.

Drawe, D. I.

Duncan, D.

Dwyer, D. D.

Eckert, R. E.

Eddlemen, L.

Ehrler, W.

Emmerich, W.

Engle, D.

Erdman, J. E.

Evans, K. E.

Evans, R. A.

Evenson, W.

Everett, R. L.

Fairbourn, M.

Ferguson R. B.

Finnell, R.

Flake, L. D.

Flinders, J. T.

Fulgham, $\mathbf{K}$.

Gartner, R. F.

Geng, S.

George, M.

Gibbens, R.

Gifford, F. G.

Godfrey, E. B.

Goetz, H.

Gonzalez, C. L.

Goodrich, S. K.

Guthery, F.

Haferkamp, M. R.

Hamilton, K.

Hanley, T.

Hanrahan, M. P.

Hansen, R. M.

Harniss, R. $O$.

Havstad, K.

Hewitt, R. E.

Hinds, $T$.

Hironaka, M.
Holechek, J. L.

Johnson, D. A.

Johnson, J.

Johnson, $M$.

Johnson, $T$.

Jolley, V. D.

Jones, M. B.

Kearl, W. G.

Kelsey, R. G.

King, R.

Kirby, D.

Klebenow, D.

Klepper, E.

Knight, R.

Kothmann, M. M.

Krueger, W. C.

Lane, L.

Lauenroth. W.

Lee, R. G.

Lewis, J. K.

Lokemoen, J.

MacCracken, J.

Malechek, J. C.

Manglitz, G. R.

McArthur, E. D.

McCaslin, $\mathbf{R}$.

McDaniel, $\mathrm{K}$.

McDonald, $B$.

Medin, D. E.

Miller, $\mathrm{R}$.

Monsen, S. B.

Morton, $\mathrm{H}$.

Moser, L. E.

Mueggler, W. F.

Murphy, A.

Murray, R. B.

Myers, R.

Neal, $D$.

Neff, E.

Nelson, D. L.

Nelson, S. D.

Nielsen, D. B.
O'Conner, G.

Ogden, $P$.

Owensby, C. E.

Paul, E. A.

Pearson, $\mathrm{H}$.

Peek, J. E.

Peterson, G. A.

Pfeiffer, G. H.

Powell, J.

Power, J. F.

Quigley, T. M.

Raguse, C. A.

Raleigh, R. D.

Rasmussen, $P$.

Reginato, $\mathbf{R}$.

Renard, $\mathbf{K}$.

Rice, R.

Richards, J. H.

Rickard, W. H.

Risser, P. G.

Rittenhouse, L.

Robbins, C.

Romm, J. M.

Roundy, B.

Rumble, $M$.

Samuel, M.

Sanders, K. D.

Schmitz, R. F.

Schrieber, H.

Schrumpf, B.

Schuman, G. E.

Scifres, C. J.

Segelquist, C. A.

Severson, $K$.

Short, H. L.

Shupe, J.

Simanton, J. R.

Sims, P. L.

Skujins, J.

Smith, L. E.

Smith, M. A.

Smoliak, S.
Sneva, F. A.

Sosebee, R. E.

Southard, A. R.

Stevens, J. E.

Stevens, R.

Stevenson, G.

Stroehlein, J. L.

Stout, D. G.

Stubbendieck, J. L.

Stuth, J. W.

Stutz, H. C.

Taylor, G. Thill, R.

Thomas, $\mathbf{S}$.

Tiedemann, A. R.

Timm, R. M.

Trilica, M. J.

Tromble,. J.

Tueller, $P$.

Uresk, D. W.

Urness, P.

Vallentine, J. F.

Varner, L. W.

Vavra, M.

Verner, J.

Vogel, K. P.

Wagestaff, F. J.

Wallace, J. D.

Waller, $\mathbf{S}$.

Welch, T. G.

West, N. E.

White, L. M.

White, R. S.

Whysong, $G$

Wiens, J. A.

Wight, J. R.

Williams, W. A.

Wilson, A. M

Winward, A. H.

Workman, J. P.

Wright, $\mathrm{H}$.

Ymamoto, $\mathrm{T}$.

Young, J. A. 